\title{
Interactive comment on "The enhancement of droplet collision by electric charges and atmospheric electric fields" by Shian Guo and Huiwen Xue
}

\section{Anonymous Referee \#3}

Received and published: 16 April 2020

A review of the manuscript acp-2019-1140 "The enhancement of droplet collision by electric charges and atmospheric electric fields" authored by Shian Guo and Huiwen Xue.

The authors numerically investigate collisions of charged cloud droplets and rain drops, accounting for influence of atmospheric electric field. For this purpose, they first calculate collision efficiency table considering gravity force, drag forces and electrical forces acting on drops in course of their interaction. Corresponding drop motion equations are formulated using superposition method and are integrated using second order RungeKutta method. Then authors solve stochastic collection equation (SCE) for 2D drop 
size distribution (DSD), where the first independent variable is drop mass and the second the drop charge. SCE is solved for various initial DSDs, charges and electric field strengths. Authors conclude that "electric field could significantly enhance the collision process" in the case when the initial DSD is given in the range of small cloud droplets. I would like to note that theory and methods used by the authors in their research are not new (all the needed references are given in the study). Nevertheless, the results obtained in the study are of interest so I recommend the manuscript for publication in ACP after major revision.

1. The English language of the manuscript is of a very low quality. Please find a way to enhance it in order to render the text more readable and comprehensible. 2. It is worth explaining in the introduction how charges appear in cloudy drops 3 . Line 123: Suddenly, the concept of "no-slip boundary conditions" appear. To explain. 4. To illustrate Eqs. (11) and (12) by a figure. To show directions of all the forces acting on drops and the velocities of the drops. 5. Is it right that appear in the Eq. (12)? 6. Line 153: actually, you integrate the system of 12 (or 8) equations 7. Line 187: Eq. (13) is the exponential distribution and not the gamma distribution. 8. How did you obtain Eq. (15) from Eq. (14)? 9. The authors should check the correctness of equations (13-15). 10. Lines 195-200. To add information about number concentration, liquid water content and charge content for all initial drop spectra. 11. Line 216: Please add the figure showing collision efficiency between cloud droplets (1-20 ÏAnm in radii), the same as in fig. 5 . It is all the more important because you obtained the maximal effect for cloud droplets. 12. Figure 6: Please, add illustrations for different collectors (say $15 \mathrm{ïA} \backsim \mathrm{m}$, and $10 \mathrm{ÏA} \backsim \mathrm{m}$ in radii) and comment them. 13. Section 5.2: Please show temporal changes of drop concentration and charge content and comment on them. How fast the charges of opposite signs compensate each other? 14. Line 288: "The relative terminal velocity term also contributes to the collection kernel, and the electric field can affect terminal velocity of small charged droplets significantly." - Please, cover this issue in more detail in the article.

Printer-friendly version

Discussion paper
Interactive

comment 
Interactive comment on Atmos. Chem. Phys. Discuss., https://doi.org/10.5194/acp-2019-1140, 2020.
ACPD

Interactive comment 